\title{
Optimization of Automated Warehouse Location Based on Genetic Algorithm
}

\author{
Wanlei Wang, Jian Gao*, Tianyi Gao and Haiting Zhao \\ Dalian Nationalities University, Dalian Liaoning, China \\ *Corresponding author
}

\begin{abstract}
The automated warehouse built in many enterprises still need through manual intervention or random allocation method for distribution of goods allocation. This distribution does not guarantee that the utilization rate of warehouse is high, especially when more types and a large number of goods, need more scientific and reasonable scheme for the storage of goods, and enhance great application value and practical significance for the competitiveness of enterprises. In order to improve the efficiency and utilization of automated warehouse, we take automated warehouse as the object to study the optimization of the storage location of goods based on the genetic algorithm in this paper.
\end{abstract}

Keywords-logistics; location assignment; genetic algorithm; automated warehouse

\section{INTRODUCTION}

Automated warehouse integrates purchasing, storage, sales, sowing, sorting and management, it is an automatic access freight system that the operator does not need to process directly, it's advantages include high utilization rate of the unit area, automatic management, labor saving, quick and accurate operation of the in and out. Since its emergence, it has quickly become the core equipment of modern logistics system, and has been widely used in industrial enterprises ${ }^{[1]}$.

The slotting optimization for automated warehouse can improve the comprehensive performance of the warehouse operation, it has become a research direction of logistics warehousing, researcher in this direction has also done a lot of research.

For example, Li Xiaoli ${ }^{[2]}$ studied the small stereoscopic warehouse divided by the size of the warehouse, aimed at the unique features of small warehouse operation, based on the sequence of single objective optimization and put forward a new strategy of warehouse location assignment; Nastasi G detailed described three popular algorithms that can handle multiobjective optimization, and discusses the results that can be achieved extensively. The genetic algorithm is applied to the optimization of the existing automated warehouse in the iron industry and this project has achieved good results. The actual structure of stereoscopic warehouse will be combined in this paper. To establish the mathematical model for the optimization problem based on the optimization principles and strategy in the location assignment and focus on the process of the genetic algorithm application to slotting optimization. By using the genetic algorithm to solve the multi-objective optimization problem, get the reasonable method for the location allocation problem.

\section{THE ESTABLISHMENT OF AUtOMATED WAREHOUSE StORAGE SPACE Optimization MODEL}

\section{A. Basic Assumptions of Mathematical Models}

Suppose there are $A$ rows of high-rise shelves in the warehouse, each row has $B$ columns and $C$ layers, a total of $A * B$ * $C$ locations. The outermost layer is the first row, and the nearest row is the first column, the bottom of the shelf is first layers. The coordinate $(x, y, z)$ represents the position of the cargo space in the $\mathrm{x}$ row $\mathrm{y}$ column $\mathrm{z}$ layer in the shelf, the $x=1$, $2 \cdots A ; y=1,2 \cdots B ; z=1,2 \ldots C$. Different types of warehouses have different shelf structure, this article is based on the general unit cargo format warehouse modeling, we make the following assumptions:

1) Suppose that the goods need to be stored in the warehouse, the volume of the goods placed in the tray is completely matched with the size of the pallet and the cargo space, and there is no situation where the cargo cannot be placed due to the size of the goods.

2) Suppose that the pallet used to load the cargo has the same size and maximum carrying weight in the general unit cargo warehouse, the cargo can be stored in different sizes within the maximum load weight range.

3) In general, united automatic warehouse space has two kinds of specifications, namely slotting length and width ratio of type 1:1 and type 5:3. To simplify the design of the model suppose the hypothesis space with the ratio of length, width and roadway width is $1: 1: 1$, and the length of the cargo is defined as $L_{0}$.

4) Suppose that the goods in the pallet are unlikely to be mixed. That is, the quality of the units of the same goods is the same as that of the same kind of goods placed in a tray.

5) Suppose the slotting optimization problem isn't relevant to stacking machine start and stop time, the fork time when it took the goods, and handling time of conveying equipment during storage and out of warehouse.

\section{B. Model Variable Definition}

$\left(x_{k}, y_{k}, z_{k}\right)$ is the corresponding coordinate of a location value, the $K(K \epsilon[1, n])$ expressed the $K$ class of goods placed in the current location. The $v_{x}, v_{y}$ and $v_{z}$ respectively represent the speed of the stacker in the $x, y$ and $z$ directions, the moving speed of a stacker in three directions is uniform and known. $\mathrm{L}_{0}$ is the height of the cell grid, $m_{k}$ is the quality of category $K$ goods, 
$n_{x y z k}$ is the number of category $K$ goods deposited $\operatorname{in}\left(x_{k}, y_{k}, z_{k}\right), P_{k}$ is the turnover rate of category $K$ goods.

\section{Determine the Slotting Optimization Mathematical Model}

\section{1) Turnover rate analysis}

The rate of turnover of goods refers to the amount of turnover of the goods in a warehouse in a year, its numerical value reflects the flow of the goods. It can be understood as the outgoing frequency of the goods. For outgoing goods with high frequency, they should be placed close to the shed port. And for the storage of the low frequency can be assigned to far away from the outlet of the storage space. This will reduce the delivery time for the goods that need to be shipped out frequently. A mathematical model is expressed as follows:

$$
f_{1}(\mathrm{x}, \mathrm{y}, \mathrm{z})=\sum_{x=1}^{A} \sum_{y=1}^{B} \sum_{z=1}^{C}\left(\frac{x_{k}}{V_{x}}+\frac{y_{k}}{V_{y}}+\frac{z_{k}}{V_{z}}\right) \times L_{0} \times P_{k}
$$

\section{2) Shelf stability analysis}

In order to avoid the stability of the shelf due to uneven force problems. It need to consider the gravity center of shelf when in slotting optimization. Arrange the heavy weight of the goods are arranged on the bottom shelf, light weight of the goods placed above the cargo space. Rely on this approach to reduce the center of gravity of the shelf and increase the stability of the overall shelf. A mathematical model is expressed as follows:

$$
\min _{2}(x, y, z)=\min \sum_{x=1}^{A} \frac{\sum_{y=1}^{B} \sum_{z=1}^{C} m_{k} \times n_{x y k} \times z \times L_{0}}{\sum_{y=1}^{B} \sum_{z=1}^{C} m_{k} \times n_{x y z k}}
$$

\section{3) Freight correlation analysis}

The relevance of the goods is based on the classification strategy and the correlation principle of the goods. The higher the correlation between the goods, the closer the storage position of the good. This can be more suitable for the situation that relevant goods need to delivery from storage in the same batch. Therefore, the goods should be placed near the central coordinates when the goods are placed, thus realize classification and stacking. The center position coordinate of $K$ goods is $\left(a_{k}, b_{k}, c_{k}\right)$.A mathematical model is expressed as follows:

$$
\begin{aligned}
& \operatorname{minf}_{3}(x, y, z)= \\
& \min \sum_{x=1}^{A} \sum_{y=1}^{B} \sum_{z=1}^{C}\left(\sqrt{\left(x_{k}-a_{k}\right)^{2}+\left(y_{k}-b_{k}\right)^{2}+\left(z_{k}-c_{k}\right)^{2}}\right)
\end{aligned}
$$

To sum up, the multi-objective mathematical model of slotting optimization is expressed as follows:

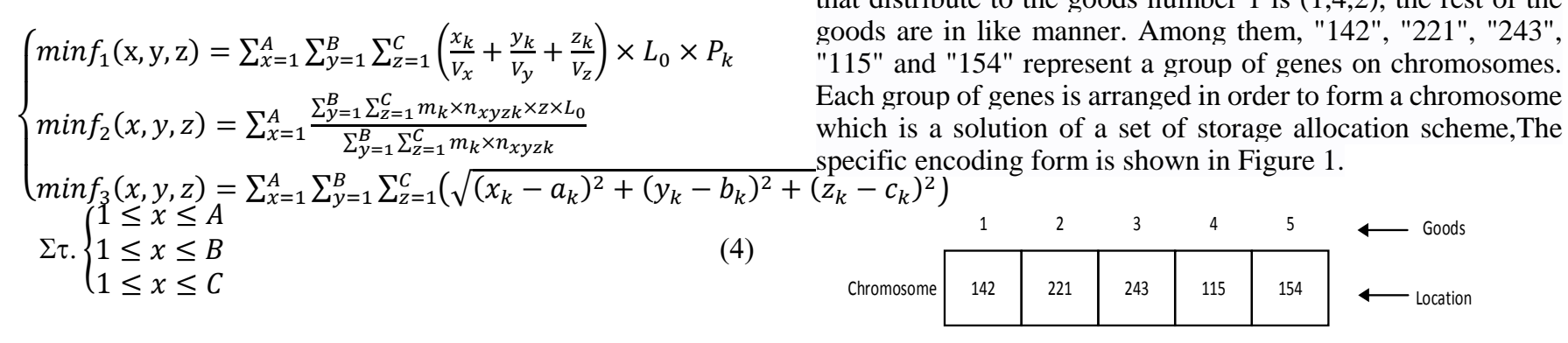

FIGURE I. SCHEMATIC DIAGRAM OF OPTIMUM CODING OF GOODS

\section{SOLVING MODEL}

\section{A. Solving Algorithm}

This paper adopts genetic algorithm to solve the objective function of the Slotting Optimization problem on Automated Warehouse. Because of the conflict and influence of each objective function, the performance improvement of a target may lead to the decrease of other target performance. This means that it is impossible to achieve optimization of multiple objectives at the same time, but only to coordinate and compromise the relation between them, so that all the targets can obtain the non-inferior optimal solution as much as possible. Therefore, the weight coefficients of the genetic algorithm are required to assign different weights to each objective function, and the model is transformed into a single objective function ${ }^{[5]}$. Model as follows:

$$
\begin{aligned}
& \operatorname{minf}(x, y, z)=w_{1} \operatorname{minf}_{1}(x, y, z)+w_{2} \min f_{2}(x, y, z) \\
& +w_{3} \operatorname{minf}_{3}(x, y, z) \\
& w_{1}+w_{2}+w_{3}=1 \\
& \text { s.t. }\left\{\begin{array}{l}
0 \leq w_{1} \leq 1 \\
0 \leq w_{2} \leq 1 \\
0 \leq w_{3} \leq 1
\end{array}\right.
\end{aligned}
$$

\section{B. Algorithm Design}

1) Coding method determination

Slotting optimization problem in this paper is a multiobjective combinatorial optimization problem which needs to find the best feasible scheme under certain restriction condition, and Integer encoding and permutation coding are more effective than other coding methods in solving multi-objective combinatorial optimization problems. Therefore, integer encoding and permutation coding are combined to encode the chromosomes of the feasible solution of the problem in this paper. It can directly show the number and location coordinates of goods in chromosomes, specific description is as follows:

TABLE I. THE GOODS NUMBER AND STORAGE LOCATION COORDINATES

\begin{tabular}{cc}
\hline goods number & Location coordinates \\
\hline 1 & $(1,4,2)$ \\
2 & $(2,2,1)$ \\
3 & $(2,4,3)$ \\
4 & $(1,1,5)$ \\
5 & $(1,5,4)$
\end{tabular}

The first row in the table represent the location coordinates that distribute to the goods number 1 is $(1,4,2)$, the rest of the 


\section{2) Initial population}

In this paper, chromosomes are randomly generated in the correct range when the initial population is set up. The size of the population affects not only the final result of optimization, but also the efficiency of the optimization process.

\section{3) Determine fitness function}

For the location of multi goal optimization model of this paper, three objective function is minimum and the function values are non-negative number, this situation can generally take the value of the objective function as the reciprocal of the fitness function. However, there is a great difference between the objective function values in this paper. It is not suitable to solve the three objective functions by weighted sum.

In order to solve the problems aforementioned, the objective functions are treated by dimensionless method In this paper,Then, the fitness function is obtained by reciprocal counting the objective function. Disposed by linear transformation in dimensional processing, the objective function can be expressed as:

$$
f_{i}^{*}=A * f_{i}+B
$$

Among them, the size of A and B is determined as follows:

$$
\left\{\begin{array}{l}
A=\frac{1}{f_{\text {imax }}-f_{\text {imin }}} \\
B=\frac{-f_{\text {imin }}}{f_{\text {imax }}-f_{\text {imin }}}
\end{array}\right.
$$

Substituting formula (7) into formula (6) can obtain:

$$
f_{i}^{*}=\frac{f_{i}-f_{\text {imin }}}{f_{\text {imax }}-f_{\text {imin }}}
$$

In the formula, $f_{i}$ represents the objective function $\mathrm{i}, f_{\text {imin }}$ and $f_{\text {imax }}$ represent the minimum and maximum values of the objective function $f_{i}$. The three objective functions Disposed by linear transformation can correspond to $(0,1)$ intervals and become dimensionless functions. Such a linear transformation does not affect the distribution of the original objective function, and does not reduce the performance of the genetic algorithm. The fitness value of each objective function can be obtained by reciprocal counting formula (8), To avoid the situation of result overflow, that is, if the denominator value is near zero, the fitness function value will tend to infinity, and we need to add 1 to the denominator. Therefore, the value of the fitness function can be expressed as:

$$
F_{i}(x, y, z)=\frac{f_{\text {imax }}-f_{\text {imin }}}{f_{i}-f_{\text {imin }}+1}
$$

Finally, the fitness function corresponding to the multi objective function can be obtained by the weight coefficient method:

\section{4) Select operation}

In this paper, random sampling method is used in the selection process, like Roulette wheel selection method to calculate selection probabilities. It is only in random ergodic selection that the choice of individuals by equal distance is required.

\section{5) Cross operation}

The crossover operation in this paper is a single point switching method

\section{6) Mutation operation}

The mutation operations chosen in this paper are discrete mutation operators. Its specific approach is: Mutating each element with a particular probability, after a series of complete operations, eventually produces a new generation.

7) Adaptive adjustment of crossover and mutation probability

In the genetic algorithm, the cross probability $P_{c}$ and the mutation probability $P_{m}$ 's value will largely affect the convergence of genetic algorithm and the proximity between the optimal solution and the real optimal solution. In this paper, the crossover probability and mutation probability can be adaptively adjusted to ensure the diversity of the group:

$$
\begin{aligned}
& \text { Cross probability: } P_{c}=\frac{1}{1+e^{\left(-k_{1} \Delta f\right)}} \\
& \text { Mutation probability: } P_{m}=1-\frac{1}{1+e^{\left(-k_{2} \Delta f\right)}}
\end{aligned}
$$

In the formula, $k_{1}$ and $k_{2}$ indicate the adjustment rate, all of which are valued at 1 . The variation of crossover probability and mutation probability is mainly based on the difference between the maximum fitness value and the average fitness of the chromosome individuals, that is : $\Delta \mathrm{f}=f_{\text {max }}-f_{\text {ave }}$.

\section{Simulation AND ANALYSis OF EXAMPLE}

Taking an automated warehouse as an example, the basic parameters of the optimization simulation are set up in table 2 .

TABLE II. BASIC PARAMETERS OF OPTIMIZATION SIMULATION

\begin{tabular}{cccc}
\hline X direction moving speed $v_{x}$ & $15 \mathrm{~m} / \mathrm{min}$ & $\begin{array}{c}\text { storage rack rows } \\
\text { number }\end{array}$ & 2 \\
\hline Y direction moving speed $v_{y}$ & $30 \mathrm{~m} / \mathrm{min}$ & $\begin{array}{c}\text { storage rack } \\
\text { columns number } \\
\text { storage rack plies } \\
\text { Z direction moving speed } v_{z}\end{array}$ & $12 \mathrm{~m} / \mathrm{min}$ \\
Goods & $0.53 \mathrm{~m}$ & $\begin{array}{c}\text { The distance } \\
\text { between each row } \\
\text { of racks } \\
\text { allocation unit length } L_{0}\end{array}$ & $0.6 \mathrm{~m}$ \\
Iterations of genetic algorithm & 100 & $\begin{array}{c}\text { Initial population } \\
\text { size }\end{array}$ & 2000 \\
\hline
\end{tabular}

At present,16 inbound goods are randomly generated, and the basic information and storage scheme are shown in table 3. 
TABLE III BASIC INFORMATION OF GOODS AND STORAGE SCHEME

\begin{tabular}{|c|c|c|c|c|c|}
\hline $\begin{array}{c}\text { Cargo } \\
\text { number }\end{array}$ & category & $\begin{array}{c}\text { Turnover } \\
\text { rate }\end{array}$ & $\begin{array}{c}\text { Total } \\
\text { mass } \\
(\mathrm{kg})\end{array}$ & $\begin{array}{c}\text { Coordinates } \\
\text { before } \\
\text { optimization }\end{array}$ & $\begin{array}{c}\text { Coordinates } \\
\text { after } \\
\text { optimization }\end{array}$ \\
\hline 1 & A & 0.15 & 12 & $(1,1,2)$ & $(2,4,1)$ \\
\hline 2 & C & 0.80 & 6 & $(1,10,5)$ & $(1,3,3)$ \\
\hline 3 & D & 0.26 & 10 & $(1,4,5)$ & $(1,3,1)$ \\
\hline 4 & C & 0.71 & 15 & $(1,6,1)$ & $(2,2,3)$ \\
\hline 5 & B & 0.42 & 1 & $(1,2,3)$ & $(2,4,3)$ \\
\hline 6 & A & 0.35 & 20 & $(2,3,4)$ & $(1,1,1)$ \\
\hline 7 & B & 0.59 & 24 & $(1,7,3)$ & $(2,3,2)$ \\
\hline 8 & B & 0.66 & 12 & $(2,6,3)$ & $(2,2,1)$ \\
\hline 9 & C & 0.77 & 15 & $(1,3,4)$ & $(1,2,2)$ \\
\hline 10 & D & 0.24 & 30 & $(2,1,3)$ & $(1,2,3)$ \\
\hline 11 & A & 0.52 & 2 & $(1,4,1)$ & $(1,1,2)$ \\
\hline 12 & B & 0.19 & 12 & $(1,7,1)$ & $(1,3,2)$ \\
\hline 13 & C & 0.82 & 16 & $(1,5,3)$ & $(2,2,4)$ \\
\hline 14 & D & 0.32 & 23 & $(1,10,1)$ & $(2,2,2)$ \\
\hline 15 & A & 0.23 & 5 & $(2,5,5)$ & $(2,3,4)$ \\
\hline 16 & D & 0.43 & 7 & $(2,8,1)$ & $(1,2,1)$ \\
\hline
\end{tabular}

In the optimization model, a simple weighted sum method is adopted to solve the multi-objective programming genetic algorithm. The weight coefficient of the optimization target is set at 0.33 , to verify the overall effect of multi-objective optimization. According to the information of initial location distribution of goods, the initial location assignment scheme is plotted in Figure 2 (different colors of diamonds represent different kinds of goods) by run the MATLAB simulation program. Can be seen in the slotting optimization before the goods stored in the warehouse is arbitrary and unreasonable.

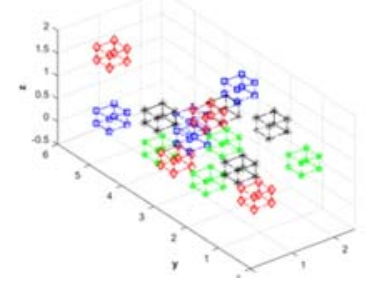

FIGURE II.THE INITIAL LOCATION LAYOUT

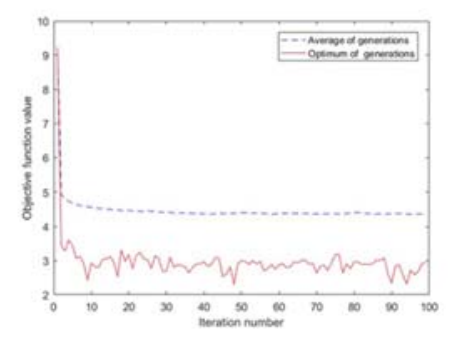

FIGURE III. OBJECTIVE FUNCTION SIMULATION RUNNING RESULT TRACKING

Considering the goods turnover rate, storage rack stability and correlation factors of the goods before completion of slotting optimization, the objective function simulation running result tracking and after the layout optimization are shown in Figure 3 and 4.

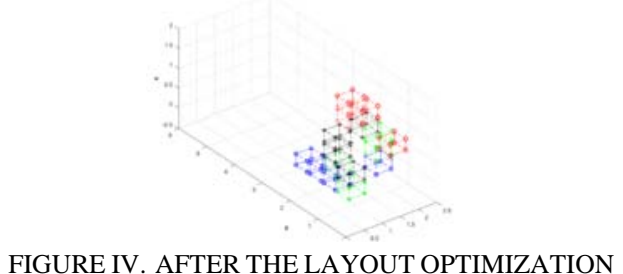

From the simulation result tracking, Figure 3 shows that the total objective function value drops from 9.1361 to 2.9126, data swings steadily as they evolve into the 15 generation. The optimized location meet the condition that put the high turnover rate of goods in the goods allocation near the outgoing stand, according to the correlation between goods and it also improves shelf stability The simulation result shows that the process of slotting optimization in large warehouse, because of the need for storage of goods in large scale, it will require a very long time if we use the traditional methods for solving optimization solution, cannot meet the need to improve the efficiency of the practical requirements. The optimization results have shown above display that genetic algorithm applied to the slotting optimization has an ideal result, can solve the mathematical model of the problem effectively. Although the global optimal solution cannot be guaranteed, the convergence rate is faster, and an approximate solution near the global optimum can be obtained in a short time. So the application of genetic algorithm to the optimization problem has great practical value.

\section{CONCLUSION}

In order to study the slotting optimization problem, this paper selected three targets include the turnover of goods, the gravity center of the storage rack and the relevance of the goods, and established slotting optimization model. The model based on the improve operating efficiency, convenient management, enhance the stability of the storage rack, also can realize the optimization of storage space. In the process of the simulation, based on genetic algorithm can effectively solve the multi-objective optimization problem, the algorithm is clear and easy to realize, for a short time, an ideal global optimum can be obtained rapidly. The problem of too long calculation time of traditional solution method is solved because of large warehouse size and large quantity of goods.

\section{ACKNOWLEDGMENT}

This research is supported by Research Foundation of Liaoning Educational Committee (L2013511) and Self-Topic Foundation of Dalian Nationalities University (DC201502010301).

\section{REFERENCES}

[1] Gu Ping. Optimization Research of automated stereoscopic warehouse [J]. science and technology outlook, 2017, 27 (6)

[2] Li Xiaoli, Liu Guizhi, Yang Wenliang. Location allocation and optimization of warehouse based on Nested Partitions Method [J]. Computer engineering and Applications ,2014, 50 (2): 242-246.

[3] Nastasi G, Colla V, Cateni S, et al. Implementation and comparison of algorithms for multi-objective optimization based on genetic algorithms applied to the management of an automated warehouse[J]. Journal of Intelligent Manufacturing, 2016:1-13. 
[4] Li Xia, Zhu Yuming. Modeling and Simulation of automated warehouse dynamic storage allocation [J]. Logistics technology, 2016, 39 (3): 131136.

[5] Deng Aimin, Cai Jia, Mao Lang. The automated warehouse slotting optimization model based on time [J]. Chinese Management Science, 2013, 21 (6): 107-112.

[6] Li Jingbo, Zhang Weiwei. Study for warehouse slotting optimization model based on Genetic algorithm [J]. Management modernization, 2016, 36 (6): 99-101.

[7]Kofler M, Beham A, Vonolfen S, et al. Modelling and optimizing storage assignment in a steel slab yard[C]// IEEE International Symposium on Logistics and Industrial Informatics. IEEE, 2012:101-106.

[8] Yao Qi, Zhou Le, Chen Baosheng, et al. Study on the loading of goods in automated warehouse based on genetic algorithm [J]. electric automation, 2016, 38 (4): 45-48.

[9] Li Xia, Zhu Yuming. Modeling and Simulation of automated warehouse dynamic storage allocation [J]. logistics technology, 2016, 39 (3): 131136. 